\title{
Image und Reputation - die Unternehmenskommunikation im Neopragmatismus
}

\author{
Jan Rommerskirchen
}

An Theorien der Kommunikation mangelt es nicht, obwohl die wissenschaftliche Beschäftigung damit noch rechtjung ist. Sie stammen von Kommunikationswissenschaftlern und-psychologen, Volkswirten und Logistikern oder Informatikern und Politikwissenschaftlern. Innerhalb des weiten Feldes der Kommunikationsforschung gibt es jedoch eine Trennlinie - die zwischen sprachphilosophischen und soziologischen Theorien. Die Theorie des "Inferentialismus" von Robert B. Brandom und sein Modell der "deontischen Kontofübrung" zeigen, wie man die Trennung zwischen Sprachphilosophie und Soziologie überwinden kann. Darüber hinaus ermöglicht der Inferentialismus eine neue Theorie der strategischen Kommunikation zwischen Unternebmen und Konsumenten. Image und Reputation eines Unternebmens können dadurch als normative Status beziehungsweise normative Einstellungen der Rezipienten von Kommunikationsakten verstanden und gezielter in die Planung der Unternebmenskommunikation integriert werden.

Schlagwörter: Kommunikationstheorie, Robert B. Brandom, Inferentialismus, deontische Kontoführung, Unternehmenskommunikation, Image, Reputation

\section{Positionen}

Vor gut einhundert Jahren verfasste Charles Horton Cooley seine Studien über das amerikanische Transportsystem und dessen ökonomische und soziale Bedeutung. Er stellte dabei fest, dass menschliche Gesellschaften wesentlich auf Austauschbeziehungen basieren und dass Kommunikation damit letztlich die Grundlage des ökonomischen und sozialen Handelns ist. Kommunikation, so Cooley, sei die Ursache für menschliche Beziehungen und Gesellschaften, für Kultur, Ökonomie und Politik: „By communication is here meant the mechanism through which human relations exist and develop - all the symbols of the mind, together with the means of conveying them through space and preserving them in time" (Cooley 1909: 61).

Mit dieser wegweisenden Definition legte Cooley den Grundstein für die weitere wissenschaftliche Beschäftigung mit dem Thema der Kommunikation, insbesondere in den USA. Hier wurden die Erforschung von Kommunikation und die daraus erwachsenden Kommunikationstheorien bald zum Erklärungsmodell für soziales Handeln im Allgemeinen und stärkten die junge Wissenschaft der Soziologie auf ihrem Weg in die institutionalisierte Forschung an den Hochschulen. Dies galt und gilt insbesondere für die Traditionslinie des Pragmatismus (griech.pragma $=$ Handlung) in der Nachfolge von John Dewey und George Herbert Mead.

In der europäischen Soziologie galt die Erforschung der Kommunikation lange Zeit als nicht anschlussfähig. Vor allem die strukturalistische Sprachwissenschaft, wie sie von Ferdinand de Saussure maßgeblich geprägt worden war, der die Praxis der Sprache, das Sprechen (parole), „als nicht theoriefähig aus dem Untersuchungsgebiet der Sprachwissenschaft aus[schloss]“ (Bucher 2004: 269), verhinderte bis in die 1960er Jahre eine wissenschaftliche Diskussion. Erst der Einzug des Konstruktivismus in die Soziologie, vor allem die wirkungsmächtige Arbeit Die gesellschaftliche Konstruktion der Wirklichkeit von Peter L. Berger und Thomas Luckmann (1969/2000), weckte das Interesse am Zusammenhang von Kommunikation und sozialem Handeln. Durch die Arbeiten von Jür- 
gen Habermas (Theorie des kommunikativen Handelns, 1981) und Niklas Luhmann (Soziale Systeme, 1984) wurde die Kommunikation bald zum Nucleus im Verständnis des sozialen Handelns und Lebens.

Die Aktualität dieses Konzepts zeigt sich auch in der Allgegenwart des Kommunikationsbegriffs: Wir sprechen von der Kommunikationsgesellschaft und besuchen Kommunikationsseminare, es gibt Kommunikationsunternehmen, Kommunikationsmedien und Kommunikationsplattformen, es gibt kommunizierende Autos, Häuser und Kühlschränke. Der Internetsuchdienst Google lieferte zu Beginn des Jahres 2011 mehr als 350 Millionen Ergebnisse für den Begriff communication und mehr als 20 Millionen für den deutschen Begriff Kommunikation.

Diese Präsenz wird auch von der Fachwelt entsprechend gewürdigt: Die Soziologen Hubert Knoblauch und Thomas Luckmann sprechen - nach dem linguistic turn in den 1980er Jahren und dem cultural turn in den 1990er Jahren - von einem communicative turn (Knoblauch/Luckmann 2000) in der Soziologie, der US-amerikanische Kommunikationswissenschaftler James Lull von einem Age of Communication (Lull 2001) und Jo Reichertz erkennt in der Kommunikationswissenschaft eine „moderne Schlüsselwissenschaft" (Reichertz 2009: 33).

Die zweite Traditionslinie, um die es hier geht, ist die Rolle der Kommunikation in der Sprachphilosophie. Auf den ersten Blick scheint es sich dabei um ein Missverständnis zu handeln: Für die Philosophie war Kommunikation immer nur das Resultat erfolgreicher Sprachbeherrschung, folglich verstanden sich die Philosophen traditionell als Analytiker der Sprache und der Relation zwischen Sprecher und Welt, nicht aber der Beziehung zwischen Sprechern.

Beispielhaft zeigt sich dies in Platons Dialog Theaitetos, in dem er die Erforschung der Wahrheit eines Sachverhalts als Akt der Kommunikation umschreibt. Im Dialog zweier Menschen soll durch Fragen und Antworten ein Sachverhalt geprüft und immer genauer beschrieben werden. Platon lässt Sokrates hierzu das berühmte Bild von der Hebammenkunst zeichnen. Wie eine Hebamme im Zusammenspiel mit der Mutter ein Kind auf die Welt bringt, so versucht Sokrates im Zusammenspiel mit seinem Gesprächspartner die Wahrheit über ein Thema ans Licht zu bringen. Letztlich - und das ist entscheidend - geht es Platon aber nicht um Kommunikation, sondern um den Sprecher und die Äußerung seiner Erkenntnis, so wie es der Hebamme um das Kind der Mutter geht. Der Kommunikationspartner ist, wie die Hebamme, nur ein Mittel zum Zweck, aber kein aktiver Partner in einem sozialen Prozess.

In der Folge war das Phänomen der Kommunikation für die meisten Sprachphilosophen, von der Antike über die Scholastik bis zur Neuzeit, obsolet, wenn es ihnen nur gelang, den einzelnen Sprechakt zu verstehen. Seinen Höhepunkt fand diese Auffassung im linguistic turn der Philosophie (vgl. Rorty 1967), im Wiener Kreis der 1920er Jahre und in der sogenannten Protokollsatzdebatte zwischen Carnap und Neurath, wonach die Probleme der Sprache und der Kommunikation endgültig durch die logische Analyse der notwendigen Protokollsätze auflösbar wären. Die Sprachphilosophie wurde damit zur Fundamentaldisziplin zumindest der Kulturwissenschaften: philosophia ancilla linguae.

Einen Ausweg aus dem zunehmenden logisch-analytischen Formalisierungszwang, den der Wiener Kreis vorgab, bot der Pragmatismus. Verkörpert wird dieser Paradigmenwechsel durch Ludwig Wittgenstein. Galt für ihn in seiner Frühphase noch: „Die Grenzen meiner Sprache bedeuten die Grenzen meiner Welt" (Wittgenstein 1921/2003, TLP 5.6), so durchbrach der Pragmatismus eben diese Grenzen eines starren sprachanalytischen Formalismus. Die Einführung der Sprachspiele in Wittgensteins Spätwerk 
führten Sprache und Kommunikation durch die Frage nach der pragmatischen Bedeutung auch in der Sprachphilosophie zusammen, so dass für ihn nunmehr galt: „Die Bedeutung eines Wortes ist sein Gebrauch in der Sprache“ (Wittgenstein 1953/2001, PU $\$ 43)$.

Die Trennung von formaler Sprache und Bedeutung wurde nachfolgend insbesondere von den Sprachphilosophen Paul Grice, John L. Austin und John R. Searle weiterentwickelt. Grice differenzierte zunächst bei der Bedeutung von Worten zwischen dem Sagen und dem Meinen (vgl. Grice 1957). Das Gesagte ist die wortwörtliche, natürliche Bedeutung eines Wortes, das Gemeinte ergibt sich aus seiner Implikatur. Die Implikatur ist jene Bedeutung der Wörter, die sich dem Hörer erst aus dem pragmatischen Kontext der spezifischen Kommunikationssituation erschließt. Die gemeinte Bedeutung der Worte des Sprechers geht über die natürliche Wortbedeutung hinaus, sie ist eine Folgerung der Interpretation des Hörers. Die Implikatur des Wortes Wende in dem Satz „Nach der Wende wurde alles besser" kann unterschiedlich sein, je nachdem, ob sich zwei reisefreudige Bürger der ehemaligen DDR unterhalten oder zwei Segler, die das gelungene Regattamanöver besprechen.

Auch für Austin und Searle steht die Bedeutung der Wörter in ihrem pragmatischen Kontext im Vordergrund. Für beide Sprachphilosophen ist Sprechen immer auch gleichbedeutend mit Handeln, und Kommunikation beruht für sie auf dem Umgang mit spezifischen Sprechakten, die innerhalb konkreter sozialer Kontexte etwas bewirken (vgl. Austin 1962 und Searle 1969). Der Erfolg oder das Scheitern der intendierten Absicht hängen in der Kommunikation nicht nur davon ab, was der Sprecher gesagt oder gemeint hat, sondern von der Verwendung des passenden Sprechaktes (vgl. Austin 1962: 163). Illokutionäre Sprechakte wie Versprechen oder Befehle bewirken eine anschließende Handlung, wenn der kommunikative Rahmen diese ermöglicht. Ein Versprechen oder ein Befehl sind Handlungen, die mit Wörtern vollzogen werden können, wenn die pragmatische Relation zwischen Sprecher und Hörer diese ermöglichen und damit die Wörter eine entsprechende Bedeutung bekommen können. Nicht jeder kann jedem alles versprechen oder befehlen, und der illokutionäre Akt entfaltet seine Handlungswirkung nur innerhalb der konstitutiven Regeln, die die Kommunikation und das soziale Handeln in der konkreten Situation leiten (vgl. Searle 1969: 30).

\section{Konflikte}

Obwohl es auch nach Wittgenstein zahlreiche Ansätze gab, die es ermöglicht hätten, Sprachphilosophie und Soziologie in einer pragmatisch ausgerichteten Forschungsrichtung zu verbinden, blieben die Bemühungen, Kommunikation zu erforschen, in den wissenschaftlichen Disziplinen zumeist getrennt: Die Soziologie beobachtete Kommunikationshandlungen in der Welt, unterstützt durch die oftmals experimentell arbeitende Kommunikationspsychologie, und die Sprachphilosophie blieb zumeist dem Sprecher und seiner Erkenntnisleistung verbunden. Die Konfliktlinie zwischen Sprachphilosophie und Soziologie verlief weiterhin zwischen Gedanke und Handlung, zwischen Sprache und Kommunikation, zwischen der intrapersonalen und der interpersonalen Perspektive der Forscher.

Zur Vermittlung zwischen Soziologie und Sprachphilosophie hat sich in den letzten Jahrzehnten das Verständnis der menschlichen Sprach- bzw. Kommunikationsentwicklung als äußerst hilfreich erwiesen. Wenn man beobachtet, wie Kinder lernen zu kommunizieren, dann versteht man viel über das Wesen von Sprache und Kommunikation und über seine Rolle im sozialen Handeln. So zeigt sich, dass Kinder beispielsweise den Umgang mit Zahlen und Mathematik schrittweise erlernen, indem sie zunächst die 
Grundrechenarten und kleine Zahlenmengen kennenlernen und dann mit der Zeit an den komplexeren Umgang mit großen und abstrakten Mengen herangeführt werden.

Ganz im Gegensatz zur Kommunikation: Kinder werden in eine hochkomplexe Welt des kommunikativen Umgangs von Menschen hineingeboren und müssen sich diese Welt durch Beobachtung und Interpretation weitgehend eigenständig erschließen. Die Welt kommuniziert ununterbrochen durch Begriffe und Sätze, durch Gestik und Mimik - und Kinder lernen, richtig zu kommunizieren, indem sie den Gebrauch von Begriffen und Sätzen, Gestik und Mimik imitieren und daraufhin durch Lob oder Tadel eine eigene kommunikative Kompetenz entwickeln.

Die ontogenetische Perspektive zeigt auf, worum es bei der Kommunikation nicht geht: Es ist nicht primär wichtig, die richtigen bzw. wahren Gedanken über etwas äußern zu können. Wenn dem so wäre, dann müsste man Sprache und Kommunikation - wie die Zahlen in der Mathematik - Schritt für Schritt erlernen. Zwar lernen Kinder zunächst einfache Begriffe, dann komplexere Sätze. Kompetenz entwickeln sie aber dadurch, dass sie den Gebrauch von Begriffen durch den Erfolg oder Misserfolg von Anschlusshandlungen verstehen.

Es ist aber auch nicht primär wichtig, richtige von falschen Handlungsformen unterscheiden zu können. Wenn Kinder zunächst richtige bzw. falsche Handlungen erlernen müssten, dann müsste es ein entsprechendes Kategoriensystem geben, dass man in der Erziehung vermitteln müsste, bevor Kinder zu handeln beginnen könnten. Auch hier entwickelt sich Kompetenz durch Handeln.

Aus der ontogenetischen Perspektive wird aber auch deutlich, was Kommunikation bedeutet: Kinder lernen zu kommunizieren, indem die Welt durch soziales Handeln vorgreift und Kinder sich hierin einfügen, d. h. indem sie kooperative Beziehungen aufbauen und die Komplexität der Kommunikation im Handlungsprozess verstehen: „Wir sind zunächst Kenner der Sprache in dem Sinn, dass wir die Sprache in vielen Fällen zu vielen Zwecken beherrschen, und das heißt, richtig im Sprechen und Verstehen ausüben können, wenigstens insoweit, als wir uns mit dem erreichten Erfolg in der Kommunikation und Kooperation mit anderen Menschen zufrieden geben (können). Dabei gibt es zunächst keine andere Richtigkeit in der Sprachbeherrschung als den jeweiligen Erfolg, wie er sich, je nach Fall verschieden, in der gemeinsamen Zufriedenheit über die glückende Kommunikation oder Kooperation zeigt“" (Kambartel/Stekeler-Weithofer 2005: 23).

\section{Robert Brandom und der Inferentialismus}

Die Komplexität des kommunikativen Handelns und die Schwierigkeiten, richtig zu handeln, hat der US-amerikanische Philosoph Robert B. Brandom in seinem ausführlichen Hauptwerk Expressive Vernunft untersucht. Brandom versucht darin, eine „Theorie der diskursiven Praxis“ (Brandom 2000: 12) zu entwickeln, die sich aus der pragmatischen Perspektive speist: „Die Idee ist, zu zeigen, welche Art des Verstehens und der Erklärungskraft aus der Art und Weise, wie wir reden, zu gewinnen ist, anstatt zu argumentieren, dass man in irgendeinem Sinne rational verpflichtet (obliged) ist, in dieser Art und Weise zu reden" (ebd., Hrvh. i. O.).

Robert Brandom stellt sich damit in die Tradition des späten Wittgensteins und will ebenfalls „die Bedeutungen sprachlicher Ausdrücke durch ihren Gebrauch“ (ebd.: 13) erklären. Konsequent folgt für Brandom daraus, dass der Gebrauch von Begriffen und damit die Praxis der Kommunikation nicht auf der Repräsentation aufbaut, sondern auf der Inferenz (vgl. ebd.: 19). Der Begriff steht nicht für etwas, wie in der langen Tradition des Repräsentationalismus, sondern er hat eine Bedeutung, weil er für die Sprecher einer 
Gemeinschaft in Relation zu anderen Begriffen steht. Daher kann ein Begriff dadurch erklärt werden, dass er in verschiedenen Schlussfolgerungen, also Inferenzen, sowohl die Position einer Prämisse als auch die Position einer Konklusion einnehmen kann. Die Bedeutung von Begriffen ergibt sich aus ihrem inferentiellen Gebrauch bzw. den inferentiellen Beziehungen, die dem Begriff als Träger des propositionalen Gehalts vom Sprecher zugewiesen werden.

Der Sprecher legt sich auf einen Begriff mit seinen inferentiellen Beziehungen fest und kann ihn als Behauptung in der diskursiven Praxis einsetzen. Die Prüfung des Gehalts und der Inferenz erfolgt, so Brandom in Anlehnung an Wilfried Sellars, in einem dialogischen Geben und Nehmen von Gründen: „Behaupten heißt Gründe angeben [...] Ansprüche erheben, deren Verfügbarkeit als Gründe für andere wesentlich für ihre assertionale Kraft ist. Behauptungen sind ihrem Wesen nach bereit, Gründe zu sein“ (ebd.: 255, Hvhg. i. O.).

Der Begriff bezieht seine Bedeutung, auf die der Sprecher sich festlegt, aus der Rolle, die der Begriff in Beziehung zu anderen Begriffen in anderen schließenden Folgerungen einnimmt. Wenn ein Sprecher sagt, dass die Stadt Essen nördlich von Köln liegt, so ergibt sich die Bedeutung des Begriffs „nördlich“ daraus, dass der Sprecher eine Vielzahl anderer Folgerungen daraus ableiten kann. So kann er sagen, dass Köln südlich von Essen liegt, dass Düsseldorf nördlich von Köln, aber südlich von Essen liegt, dass Ratingen nördlich von Köln und Düsseldorf, aber südlich von Essen liegt et cetera.

Von wesentlicher Bedeutung für die pragmatische Perspektive Brandoms ist dabei die normative Selbstverpflichtung der Akteure. Kommunikation und soziales Handeln beruhen demnach auf der Anerkennung von Regeln (Normen) und sozialen Rahmenbedingungen (Frames), also einer gegebenen und den Akteuren bekannten sozialen Ordnung. Talcott Parsons, Harold Garfinkel und Jeff Coulter haben diese pragmatische Vorbedingung erfolgreicher Kommunikation detailliert ausgearbeitet: „Even if we feel secure in our private moments, once we make public disclosure of our thoughts, understandings, intentions, recollections, perceptions, motives and sensations, we place ourselves within a social orbit of appraisal and judgement; our subjectivity becomes analyzable within a shared frame of reference" (Coulter 1979: 107).

Brandom macht nochmals klar, worum es bei der Anerkennung einer sozialen Ordnung (frame of reference) geht: um normative Selbstverpflichtung. Soziales Handeln geschieht nicht wie bei Tieren instinktiv oder aus einer natürlichen Notwendigkeit heraus, sondern weil Menschen als vernünftige Wesen freiwillig einer Regel beziehungsweise einer Norm folgen. Die Autorität einer Regel beruht darauf, dass „wir sie als für uns verbindlich anerkennen. Unsere Würde als Vernunftwesen besteht gerade darin, dass wir uns nur Regeln unterwerfen, die wir billigen, die wir frei gewählt haben (wie Odysseus angesichts der Sirenen), uns selbst zu binden“ (Brandom 2000: 99, Hvhg. i. O.).

Brandom stellt sich hier in die Tradition Immanuel Kants: Für beide ist die praktische Vernunft des Menschen essenziell mit der Fähigkeit zur Selbstgesetzgebung verknüpft. Das Handeln nach Regeln ist daher für Kant und Brandom der Nachweis der Autonomie eines freien und vernünftigen Menschen. Man nimmt vielleicht instinktiv wahr, dass jemand den Raum betritt, und man atmet, weil es biologisch notwendig ist. Dass Menschen sozial handeln, also auf den anderen Menschen zugehen und ihm die Hand reichen, ist jedoch ein autonomer - vernünftiger und freier - Entschluss, dem normativ Gebotenen zu folgen. Autonome Wesen entschließen sich voluntativ zu dieser sozialen Handlung, es sind weder Instinkte noch eine Notwendigkeit, die den Menschen dabei antreiben. 
Wir befolgen daher im sozialen Handeln freiwillig eine Regel bzw. folgen einem normativen Sollen, dem wir uns als selbst-gesetzgebende Wesen verbunden fühlen. Menschen nehmen damit einen normativen Status ein, und „die Normen, die für uns als rationale Wesen verbindlich sind, [werden] durch unsere praktischen Einstellungen und Handlungen instituiert. Sie sind das, was wir zum Fest mitbringen“ (Brandom 2000: 101). Das Fest ist gleichsam die Selbstverpflichtung der Akteure zur Einhaltung der Regeln in der Kommunikation, die Selbstbindung an den normativen Status und damit die Errichtung einer kollektiven sozialen Ordnung. Ob man „bereit ist, diesen Regeln zu folgen, zeigt sich in der Kommunikation selbst. Das kommunikative Handeln schafft so Verlässlichkeit" (Reichertz 2009: 233, Hvhg. i. O.) und „Handlungssicherheit" (ebd.: 228) für alle Beteiligten auf der Grundlage normativer Status.

Dieser normative Status, in den sich die Akteure begeben, zielt ab auf Richtigkeit und Angemessenheit im sozialen Handeln und damit auf den Aufbau einer kooperativen Beziebung durch Kommunikation. Wenn beide Akteure sich jeweils zu einem individuellen normativen Status verpflichten, der in Bezug auf die soziale Handlung wesentlich übereinstimmt, dann bezeichnet Brandom den dadurch erreichten gemeinsamen Status als deontischen Status. Abgeleitet vom altgriechischen ,deon', das Seinsollende, entwirft Brandom damit das Konzept einer sozialen Beziehung, die beide Akteure umfasst und in der beide Akteure dennoch jeder für sich einen normativen Status der Selbstverpflichtung einnehmen müssen. Der Akt der Kommunikation wird zum gemeinsamen Fest durch den verbindenden deontischen Status.

Der wesentliche Gedanke hierbei ist, dass der deontische Status nicht etwa von einem der Akteure alleine etabliert werden kann. Es reicht nicht, dass ein Akteur sich normativ verpflichtet und davon ausgeht, dass der andere den gleichen Schritt vollzieht. Kommunikation ist für Brandom eine soziale Praktik, weil sie - neben der normativen Selbstverpflichtung - die voluntative Anerkennung des Anderen als Kommunikationspartner einfordert. Diese Beziehung des deontischen Status zwischen Akteuren entsteht mit anderen Worten, wenn „es den Beteiligten gelingt, füreinander wichtig zu werden“ (Brandom 2000: 233, Hvhg. i. O.).

Diese wechselseitige Anerkennung erfolgt durch das dialogische Geben und Nehmen von Gründen. Unser Gegenüber kann Gründe für unsere Behauptungen und Handlungen einfordern und verstehen, warum wir dies behaupten oder jenes tun. Beispielsweise erfolgt die Begrüßung per Handschlag mit der rechten Hand innerhalb einer sozialen Ordnung, die beide Akteure kennen und anerkennen müssen. Ebenso könnten die Akteure dann auch klären, warum einer von ihnen die linke Hand zur Begrüßung reicht und nicht die verletzte rechte.

Beide Akteure können folglich ihr soziales Handeln einerseits mit der Befolgung von Normen begründen und andererseits verstehen, dass es normative Grundlagen sind, die ihr Handeln begründen. Dadurch dass die Akteure Gründe für ihr Handeln geben und diese verstehen, begeben sie sich in den „Raum der Gründe“ (ebd.: 37). Der Raum der Gründe ist der Handlungsrahmen, innerhalb dessen sich die Akteure bewegen - hier bauen sie Beziehungen auf, die als deontische Status Gemeinschaftlichkeit und ein Miteinander in der Kommunikation begründen.

\section{Kontoführung als Kommunikationsmodell}

Kommunikation ist für Robert Brandom dann erfolgreich und andauernd, wenn beide Sprecher die normativen Regeln einhalten und so den deontischen Status aufrechterhalten: „Die deontischen Status der Festlegung und Berechtigung sind formal definiert 
durch die Art, wie sie dazu dienen, die Züge zu überwachen, die im Spiel des Gebens und Verlangens von Gründen gemacht werden“ (ebd.: 275).

Dieses „Spiel des Gebens und Verlangens von Gründen“ vergleicht Brandom mit einem Baseballspiel. Beim Baseballspiel gibt es Regeln, an die sich alle Mitspieler halten müssen. Wie das Spiel verlaufen ist und wer welche Regeln erfolgreich eingehalten oder nicht eingehalten hat, lässt sich leicht am Punktestand erkennen. Die Anzahl der Spielzüge („runs“), erfolgreicher („strike“) und misslungener („out“) Spielzüge lassen sich am Punktestand nachvollziehen. Auf den Diskurs übertragen kann man „sich die Entwicklung eines sprachlichen Austauschs oder Gesprächs so vorstellen [...], dass sie impliziten Normen unterworfen ist, die (vom Theoretiker) als Punktestandfunktion explizit gemacht werden können“ (ebd.: 273).

Wie ein Schiedsrichter das Baseballspiel beobachtet und bewertet, so kann der Beobachter eines Diskurses das Geben und Verlangen von Gründen bewerten. Der Diskurs besteht aus Festlegungen und Berechtigungen eines Sprechers, die wiederum durch Festlegungen und Berechtigungen des anderen Sprechers weitergeführt werden, die wiederum durch Festlegungen und Berechtigungen des ersten Sprechers weitergeführt werden usw.

Der Grundgedanke, Kommunikation mit einem Spiel zu vergleichen, in dem die Akteure Punkte für regelkonformes Handeln erzielen, stammt von David Lewis (1983). Robert Brandom baut diesen Gedanken zu seinem zentralen Konzept der deontischen Kontoführung aus. Jeder Sprechakt im Diskurs ist ein Zug im Spiel. Ob es ein erfolgreicher Zug ist, hängt davon ab, ob der Sprecher den richtigen Zug gemacht hat - das heißt, ob die Festlegung und die Berechtigung, die er mit seinem Sprechakt vollzogen hat, an der jeweiligen Stelle des Gesprächs angebracht und regelkonform sind. Die „Signifikanz eines Sprechaktes“ in der Kommunikationshandlung zeigt sich, so Brandom, darin, wie sich der aktuelle Punktestand „auf die Angemessenheit des Sprechaktes auswirkt und wie der Vollzug dieses Sprechaktes wiederum den Kontostand beeinflusst“" (Brandom 2000: 274).

Dabei wird folglich nicht nur der jeweils aktuelle Sprechakt/Spielzug bewertet, sondern immer auch sein „kommunikativer Wert“ für den normativen Status des Sprechers und für den deontischen Status der Akteure. Der Punktestand spiegelt insofern einen Status zwischen der (gemeinsamen) Vergangenheit und der (erwarteten) Zukunft der Akteure, er ist immer auch retrospektiv und auch prospektiv. Beide Akteure blicken auf den Sprechakt und seinen unterstellten Gehalt deshalb sowohl flussaufwärts auf das Erlebte, das sie bis zu diesem Punkt gebracht hat, als auch flussabwärts auf das, was aus der Kommunikation resultieren wird: „Downstream, they must have inferential consequences, commitment to which is entailed by commitment to the original content. Upstream, they must have inferential antecedents, relations to contents that can serve as premises from which entitlement to the original content can be inherited" (Brandom 2008: 114).

Im Anschluss an den späten Ludwig Wittgenstein, Wilfried Sellars und David Lewis formuliert Brandom die Kommunikationshandlung ebenfalls als ein bedeutungsgenerierendes Sprachspiel. Im Unterschied zu den Genannten steht bei Brandom aber der Begriff und sein propositionaler Gehalt nicht mehr als Repräsentant für etwas. Der Inferentialismus betrachtet Kommunikation konsequent als soziale Praxis der voluntativen Verpflichtung beider Akteure durch Festlegungen und Behauptungen in der Kommunikationshandlung, denn ,in jedem Stadium hängt das, was man tun darf oder wozu man verpflichtet ist, vom Kontostand ab, und das gleiche gilt für die Folgen, die dieses Tun für den Kontostand hat" (Brandom 2000: 274). 
Im Konzept der deontischen Kontoführung bewirkt jeder Sprechakt in einer Kommunikationshandlung deshalb zwei Veränderungen: Der eine Punktestand beschreibt den normativen Status des Gegenübers, der andere Punktestand den deontischen Status der Beziehung zwischen den Akteuren. Jeder Akteur führt demzufolge in der Kommunikation zwei Konten, von denen eines die Behauptungen des Sprechers bewertet, ein anderes die Festlegungen und inferentiellen Verweise, die daraus folgen: „Wechselseitiges Verstehen und Kommunikation hängen davon ab, dass Gesprächspartner in der Lage sind, zweierlei Kontenbücher zu führen, dass sie sich zwischen dem Standpunkt von Sprecher und Hörer hin- und herbewegen können, während sie sich den Überblick darüber verschaffen, welche doxastischen, substitutionalen und expressiven Festlegungen von den Beteiligten eingegangen und welche zugewiesen werden“ (ebd.: 818).

Brandom versteht daher die "diskursive Praxis als eine von Grund auf soziale Praxis“ (ebd..: 691, Hvhg. i. O.), weil sie wesentlich auf der Interpretation von Festlegungen und Behauptungen in Sprechakten beruht, die die doppelte deontische Kontoführung der Akteure implizit beeinflusst: „Die deontische Kontoführung ist die Form des Verstehens, die der Kommunikation innewohnt. Sie ist eine Art des Interpretierens, aber es handelt sich um eine implizite, praktische Interpretation und nicht um eine explizite theoretische Hypothesenbildung“ (ebd.: 707).

Mit seinem Konzept der deontischen Kontoführung zerstört Robert Brandom eine tragende Säule der traditionellen Vorstellung von Kommunikation. Seit Platon besteht die zentrale Aufgabe von Kommunikation in der Übermittlung, d. h. der Sender verfügt über ein Wissen, das er durch den Sprechakt transportieren kann. Nach erfolgter Kommunikation ist auch der Empfänger im Besitz dieses Wissens, wenn er die Botschaft verstanden hat. Sender und Empfänger teilen dann ein gemeinsames Wissen über die Welt.

Für Brandom erfüllt die Kommunikationshandlung eine gänzlich andere Aufgabe, denn für ihn geht es nicht um den Austausch von Informationen und das „Verstehen“ des repräsentationalen Gehaltes von Begriffen in der klassischen Form des „Anknipsen[s] eines cartesianischen Lichts“ (ebd.: 193), sondern um Kooperation und gemeinsames soziales Handeln durch die implizite Kontoführung und „praktische Beherrschung einer bestimmten Art inferentiell gegliederten Tuns" (ebd., Hvhg. i. O.) in der Kommunikation: „Das etablierte Modell der Kommunikation als gemeinsamer Besitz von etwas wird fallengelassen zugunsten [...] eines Modells der Kommunikation als einer Art von Kooperation in der Praxis. Was Sprecher und Zuhörer teilen, ist nicht ein Gehalt-als-Funktion, sondern eine Kontoführungs-Praxis“ (ebd.: 676, Hvhg. i. O.).

Damit geht Brandom auch über Niklas Luhmann hinaus. Für Luhmann ist Kommunikation - um hier nur eine gängige Lesart aufzugreifen - die Einheit von Information, Mitteilung und Verstehen (vgl. Ortmann 2011: 359). Das Konzept der deontischen Kontoführung gibt der Kommunikation ihre performative Macht zurück, indem es die Wirkung von Sprechakten auf die soziale Praxis betont und damit die sprachphilosophischen Ansätze von Grice, Austin und Searle aufnimmt. Für Brandom ist Kommunikation mehr als Information, Mitteilung und Verstehen - ihm geht es insbesondere um die Wirkung von Sprechakten auf den Sprecher (beispielsweise in der Selbstbindung beim Sprechakt des Behauptens), den Hörer (beim Sprechakt des Versprechens) und die soziale Beziehung (in der wechselseitigen Anerkennung der Akteure).

\section{Der Neopragmatismus und die Folgen}

Mit dem Entwurf seines neopragmatischen Kommunikationsmodells erweitert Robert Brandom das Verständnis von Kommunikation als soziales Handeln um zwei wesent- 
liche neue Gedanken: Auf der intrapersonellen Ebene setzt Brandom beim normativen Status der Akteure an, die wechselseitig Konten füreinander führen. Auf der interpersonellen Ebene erweitert er diesen Grundgedanken um einen deontischen Status, der die soziale Beziehung der Akteure durch ein zweites Konto beschreibt. Der deontische Status drückt jenes Maß an Handlungssicherheit aus, das die Akteure durch Kommunikation und soziales Handeln miteinander gewinnen.

Durch den deontischen Status und die deontische Kontoführung erweitert Brandom den Blickwinkel auf die soziale Praxis des Diskurses, es geht ihm nicht nur um den Sprechakt und seine Folgen, sondern um den Aufbau einer sozialen Beziehung durch Kommunikation unter Einbeziehung der doppelten Kontoführung für den normativen Status des Sprechers einerseits und für die soziale Tragfähigkeit des Diskurses andererseits.

Mit dem Inferentialismus baut Robert Brandom insofern eine Brücke zwischen der intrapersonalen und der interpersonalen Perspektive, aus der die Sprachphilosophie einerseits, die Soziologie andererseits auf die Kommunikationshandlung blicken. Damit bietet er eine gemeinsame, vermittelnde Theorie an, die zwischen Gedanke und Handlung, zwischen Sprache und Kommunikation steht.

Seit dem Erscheinen seines Hauptwerks Making it Explicit im Jahr 1994 bzw. der deutschen Übersetzung Expressive Vernunft im Jahr 2000 wurde diese Brückenfunktion jedoch nur wenig beachtet. Zwar lobte Jürgen Habermas den Inferentialismus als Meilenstein der theoretischen Philosophie, vergleichbar mit John Rawls' Theorie der Gerechtigkeit für die praktische Philosophie unserer Zeit (Habermas 2004: 138), dennoch blieb Brandoms Arbeit in Habermas' Werk ohne erkennbare Wirkung.

Um dennoch eine kleine Auswahl seiner Rezipienten im deutschsprachigen Raum zu nennen, sei hier von Seiten der Philosophie Sebastian Knell erwähnt, der sich in einer ausführlichen Analyse mit der Sprachgebundenheit intentionaler Zustände beschäftigt hat (vgl. Knell 2004), sowie Herbert Schnädelbach, der sich in einem Artikel überaus kritisch mit Robert Brandom und dem Modell der deontischen Kontoführung auseinandergesetzt hat (vgl. Schnädelbach 2004). Von Seiten der Soziologie sei hier auf Tanja Pritzlaff verwiesen, die den Inferentialismus zur Grundlage ihrer entscheidungstheoretischen Arbeit gemacht hat (vgl. Pritzlaff 2006). Lediglich der Essener Kommunikationswissenschaftler Jo Reichertz hat bislang den neopragmatischen Entwurf von Robert Brandom in seine aktuelle Forschung einbezogen (vgl. Reichertz 2009: 232ff.).

Welche praktischen Konsequenzen sich aus dem Kontoführungsmodell für prekäre Formen der Kommunikation, beispielsweise die interkulturelle Kommunikation oder die polizeiliche Vernehmung von Beschuldigten ergeben, wurde bereits an anderer Stelle untersucht (vgl. Rommerskirchen 2011). Als „Brückenbauer“ zwischen den Fokussen Gedanke und Handlung, zwischen Sprachphilosophie und Soziologie fanden Robert Brandom und der Inferentialismus bislang jedoch zu wenig Beachtung. Dabei können die Grundgedanken eines deontischen Status zwischen den Akteuren und der doppelten Kontoführung für die Erforschung der personalen Kommunikation, aber auch der Unternehmenskommunikation, durchaus fruchtbaren Einfluss nehmen.

\section{Image und Reputation}

Das Feld der Unternehmenskommunikation grenzt an viele benachbarte akademische Terroirs, beispielsweise die Volks- und Betriebswirtschaft, die (Werbe-)Psychologie, die Kommunikations- und Sozialwissenschaften. Eine der interessantesten Grundsatzfragen auf diesem interdisziplinären Feld betrifft den Gegenstand der Kommunikation: Was wird bei der Unternehmenskommunikation eigentlich vermittelt? Oder sprach- 
philosophisch ausgedrückt: Was ist der propositionale Gehalt in der Unternehmenskommunikation?

Unabhängig davon, ob wir Teilbereiche wie Marketing- oder PR-Aktivitäten betrachten, erzeugen die Unternehmen Kommunikationsangebote, in denen der Wert eines Produktes beziehungsweise des dahinterstehenden Produzenten vermittelt wird. Da die Rezipienten zumeist über keine konkreten Erfahrungen mit dem Produkt oder dem Unternehmen verfügen, findet die Kommunikation „im Fernbereich“ (Zerfaß 2004: 127) statt. Die Grundlage der kommunikativen Beziehung zwischen dem Sender (dem Unternehmen) und den Empfängern (den Konsumenten) bezeichnet Ansgar Zerfaß als soziales Vertrauen: „Es beruht auf dem Glauben in die Zuverlässigkeit der beteiligten Akteure und der in Anspruch genommenen Strukturen. Man vertraut darauf, daß Personen bzw. korporative Akteure bestimmte positive und negative Eigenschaften aufweisen (Akteursvertrauen) und die in Anspruch genommenen Handlungsweisen [...] die ihnen zugedachten Funktionen erfüllen (Strukturvertrauen). Daraus leitet sich dann die Zuversicht ab, daß konkrete Interaktionen gelingen“ (ebd.: 126).

Den propositionalen Gehalt der Unternehmenskommunikation kann man folglich auch als soziales Vertrauen auffassen - eine Annahme, die jedoch im Widerspruch zur Theorie des Konsumenten als rationalem Akteur steht. Die Prämisse vom Homo oeconomicus sah im Konsumenten primär den rational kalkulierenden und aufgeklärten Akteur, Werbung und Public Relations dienten ihnen primär der Information. Mittlerweile ist deutlich geworden, dass diese Grundannahme nur für einen Teil der Konsumenten zutrifft, eben jene, die sich für den Gegenstand des Informationsangebotes aktiv interessieren, die dem Konsumobjekt eine besonders intensive Beteiligung beziehungsweise Aktiviertheit entgegenbringen, das sogenannte „hohe Involvement“. Volker Trommsdorff betont für diesen Bereich jedoch, dass es „eher die Ausnahme als die Regel“ sei (Trommsdorff 2009: 48). Er definiert Involvement als „Aktivierungsgrad bzw. die Motivstärke zur objektgerichteten Informationssuche, -aufnahme, -verarbeitung und -speicherung “ (ebd.: 49) und unterscheidet zwischen hohem und niedrigem Involvement, das sich durch mehrdimensionale Involvement-Determinanten in der Relation zwischen Konsument und Objekt beschreiben lässt.

In einer Konsumwelt, in der Unternehmen und Produkte zunehmend austauschbar sind und weder einen exklusiven Nutzen- noch einen eindeutigen Preisvorteil mit sich bringen, beherrscht das niedrige Involvement das Konsumentenverhalten. Eine Aktivierung der Konsumenten kann dann nur über die Symbolisierung des Produktes (durch Werbung), des Anliegens (durch Public Relations) oder des Unternehmens (durch Branding) erfolgen. Ein Ansatz, den bereits Edward Bernays, auf den Schultern von Sigmund Freud und Gustave Le Bon, in den 1920er Jahren formuliert hat: „Eine Ware wird nicht wegen ihres spezifischen Werts oder wegen ihres Nutzens begehrt, sondern weil sie als Symbol für etwas anderes steht“ (Bernays 2009: 52). Als Symbol erhält das beworbene Produkt, der Gegenstand der PR-Kampagne oder die Unternehmensmarke einen „kommunikativen Zusatznutzen“, der ihm den Zugang zur „Differenzierungsfunktion“ und zur „Identifikationsfunktion“ verschafft (Schmid/Lyczek 2008: 46). Differenzierungsund Identifikationsfunktion machen aus dem austauschbaren Objekt dann ein Symbol mit konnotativer Bedeutung, dem auf Seiten der Konsumenten hohes Involvement entgegengebracht werden kann. Voraussetzung hierfür ist jedoch die kommunikative Bedeutungsvermittlung vom Akteur zum Konsumenten: „Die symbolische Seite eines Produkts bedarf also einer kommunikativen Erzeugung" (ebd.: 47). Das Produkt der Unternehmenskommunikation ist dann die konnotative Bedeutung des Symbols - eine Vorstellung, ein Image. 
Was ein Image ist, ist aber nicht immer eindeutig und klar. Trommsdorff definiert ein Image abstrakt als „mehrdimensionale und ganzheitliche Grundlage der Einstellung einer Zielgruppe zum Gegenstand. Es besteht aus mehr oder weniger wertenden Eindrücken von der Marke, die zu einem ganzheitlichen ,Bild' verbunden sind“ (Trommsdorff 2009: 155). Schmid/Lyczek differenzieren zwischen Image und Reputation. Für sie ist das Image die individuelle, mentale Repräsentation des Objekts, ein „Vorstellungsbild, das verbunden ist mit Emotionen (Body Feelings), kognitiven Wissensinhalten und Handlungswissen sowie mit spezifischen motivationalen Antrieben" (Schmid/ Lyczek 2008: 52). Die Reputation des Objekts beschreiben sie als „die aggregierte Gesamtheit vorhandener Images eines Produktes oder einer Unternehmung bei einer gegebenen Gemeinschaft. Der Einzelne weiss, dass seine Mitmenschen zum gleichen Gegenstand ebenfalls Bilder und Gedanken besitzen. Das, was die Anderen über ein Produkt denken, das Image, welches sich die Gemeinschaft bildet, macht aus, was mit dem Begriff der Reputation angesprochen ist. Während Image das bezeichnet, was ich von einem Produkt denke, ist Reputation das, was man (die Gruppe, die Anderen) von einem Produkt oder Unternehmen denkt" (ebd.: 54).

Bemerkenswert hierbei ist jedoch die definitorische Unschärfe, mit der so zentrale Begrifflichkeiten wie Image und Reputation von den Autoren eingesetzt werden. Sie verorten die Quelle des Images beziehungsweise der Reputation in gemeinsamen Einstellungen einer Zielgruppe oder einer Art kollektiven Wissens der Gruppe. Diese Annahme führt jedoch zwangsläufig zu einer Idealisierung von Images, sie verlagert das Symbol in eine Art platonisches Ideenreich. Gänzlich unklar bleibt dann, wie diese gemeinsamen Vorstellungen entstehen, was ihr Inhalt ist und wie sie verändert werden können. Ebenso unklar bleibt, wer in welchem Maße Zugang zu beziehungsweise Anteil an diesen gemeinsamen Vorstellungen hat.

Dabei ist ein gutes Image weitaus mehr als ein „nice to have“. Selbstverständlich spielten und spielen Bekanntheit und Images bei börsennotierten Unternehmen immer eine wichtige Rolle bei der Erzielung einer maximalen Marktkapitalisierung; bei entsprechend negativer Einschätzung schwindet das Vertrauen der Investoren und in der Folge der Unternehmenswert an der Börse (vgl. Untergrabner/Ehrenhöfer 2004). Als sogenannte immaterielle Werte wurden Bekanntheit und Images durch die Einführung neuer Rechnungslegungsvorschriften gemäß der International Accounting Standards (IAS) zudem in den Mittelpunkt finanzwirtschaftlicher Betrachtungen gerückt (vgl. Horvart/Möller 2004). Ansgar Zerfaß weist eindringlich darauf hin, dass ein positives Rating - auch durch die geänderten Rahmenbedingungen für die Finanzierung von Unternehmen (Basel II) - „unmittelbare Auswirkungen auf die Refinanzierungskosten und damit auf die allgemeine Kostenstruktur der Unternehmen" hat (Zerfaß 2004: 395). Auch Schmid/Lyczek stellen fest, dass Image und Reputation als „wichtige Einflussgrößen des Stakeholder-Verhaltens [...] einen ökonomischen Wert auf[weisen], sie sind einkommenswirksam und haben daher Kapitalcharakter" (Schmid/Lyczek 2008: 133). Und auch Matthias Buck von der Deutschen Bank stellt fest, dass es bereits heute üblich ist, die Reputation mithilfe monatlich ermittelter Indexes zu erfassen, und fordert, das Thema Reputation zur Chefsache zu machen: „Es muss der unumstößliche Grundsatz gelten, dass kein Geschäft es wert ist, die Reputation und Glaubwürdigkeit des Instituts aufs Spiel zu setzen“ (Buck 2011).

Mit der Einführung von Basel III im Jahre 2013 wird die Unternehmenskommunikation noch stärkere Beachtung finden, die weichen Faktoren wie Kommunikationsarbeit und Reputation „spielen bei dem Rating oft eine wesentliche Rolle. Sie können bis zu 50 \% der Klassifizierungsmerkmalsgewichtung ausmachen“ (Müller 2011: 9) und da- 
mit entscheidend auf die Kapitalversorgung eines Unternehmens einwirken. Die Reputation hat folglich einen maßgeblichen Einfluss auf den Wert eines Unternehmens und damit auch auf seinen ökonomischen Erfolg - umso wesentlicher ist eine begriffliche und konzeptionelle Klärung, was Images und Reputation bezeichnen.

\section{Einstellungen und Milieus}

Um diese begriffliche Klärung nun im Inferentialismus zu verorten, zunächst ein Blick auf Brandoms Position. Robert Brandom vertritt eine stark nominalistische Position. Für ihn gibt es, wie bereits dargestellt, letztlich nur doxastische Festlegungen, die von Individuen als Behauptungen geäußert werden können (Brandom 2000: 240). Jeglichen Zugriff auf kollektive, objektive Einstellungen lehnt Brandom als unzulässige Übertragung von individuellem Wissen auf eine „Wir“-Behauptung ab: „Die Identifikation von Objektivität mit derart verstandener Intersubjektivität ist insofern mangelhaft, als sie der Möglichkeit des Irrtums seitens der privilegierten Perspektive keinen Platz einräumen kann; was die Gemeinschaft für richtig hält, ist richtig“ (ebd.: 831). Die Frage ist dann jedoch, wie Konzeptionen über Images und Reputation im Inferentialismus entstehen und wie sie ihre Wirkung im deontischen Kontoführungsmodell entfalten können.

Brandoms Kritik an der Objektivität gemeinsamer Einstellungen ist erkenntnistheoretischer Natur: Wahrheit ist für ihn eine diskursive Definition (vgl. ebd.: 832), sie kann nur durch wechselseitige Festlegungen und Behauptungen definiert und geprüft werden und gilt nur für diese pragmatische, lokale Perspektive, aus der die Diskursteilnehmer auf die Welt blicken. Eine globale Perspektive mit objektivem und autoritärem Wahrheitsanspruch gibt es für Brandom nicht: „Es gibt nur die tatsächliche Praxis des Prüfens, wer im Einzelfall die besseren Gründe hat. Die soziale Metaphysik des Behauptens entscheidet die Frage, was es für eine Behauptung bedeutet, wahr zu sein, indem sie klärt, was man tut, wenn man sie für wahr hält. Sie entscheidet nicht darüber, welche Behauptungen wirklich wahr sind - welche richtigerweise für wahr gehalten werden." (ebd.: 834). Niemand kann im inferentiellen Diskurs eine Vogelperspektive einnehmen und den „Kampf der konkurrierenden Behauptung“ (ebd.) Kraft seiner Autorität entscheiden.

Was bleibt, sind Zuschreibungen - und an dieser Stelle wechselt Brandom von der Beschreibung der Ich-Du-Beziehung in der interpersonellen Kommunikation zur IchWir-Relation. Als Mitglied einer sozialen Gemeinschaft verfügen die Akteure nicht nur über einen normativen Status, sondern ebenfalls über normative Einstellungen. Es sind diese normativen Einstellungen (im Original: „stances“), die den Akteur mit seiner Gemeinschaft verbinden: „In diesem Sinne einer von uns zu sein, heißt, Subjekt normativer Einstellungen zu sein, heißt, Richtigkeiten und Unrichtigkeiten von Verhaltensweisen anerkennen zu können, heißt, eine Performanz als richtig oder unrichtig behandeln zu können" (ebd.: 75).

In Bezug auf die Performanz ist es also der normative Status, der beurteilt wird, und die normative Einstellung, die beurteilt. Der Status ist eine Folge der Einstellung, sie geht ihm logisch voraus. Die Performanz eines Akteurs wird demzufolge von der Gemeinschaft als richtiges Handeln positiv sanktioniert, als falsches Handeln negativ. Die Gründe und Normen, die hinter der Sanktionierung der Performanz stehen, sind die individuellen und sozialisierten normativen Einstellungen der einzelnen Mitglieder der Gemeinschaft, die die Performanz beurteilen.

Dieses Urteil ist jedoch kein kollektiver Akt. Brandom wendet sich ausdrücklich gegen Aussagen von Kripke und Wright wie „was die Gemeinschaft als richtig ansieht“ oder „was die Gemeinschaft billigt“ und weist explizit darauf hin, dass die Zu- 
stimmung und Ablehnung von Performanzen grundsätzlich eine subjektive, akteurgebundene Tätigkeit ist (ebd.: 83). Der Verweis auf einen Smith'schen impartial spectator ist für Brandom lediglich eine „façon de parler“ und ein „Orientierungsfehler“ (ebd.: 84).

Das Beurteilen einer Handlung meint, dass eine Person aufgrund ihres normativen Status das Handeln einer anderen Person und ihres zugewiesenen normativen Status beurteilt - vor dem Hintergrund einer normativen Einstellung der beurteilenden Person, die sie der Gemeinschaft zuweist: „Beurteilen, Billigen usw. sind alles Dinge, die wir Einzelne tun und einander zuerkennen, und wodurch eine Gemeinschaft, ein Wir entsteht“ (ebd.). Dieses Wir ist demzufolge eine Konsequenz aus der Zuerkennung einer normativen Einstellung an eine Gruppe von Personen. Damit wird aus der Ich-Du-Beziehung, aus der sich auf der Grundlage zweier kongruenter normativer Status ein deontischer Status bilden kann, eine Ich-Wir-Beziehung gefolgert und konstruiert.

Für die Unternehmenskommunikation folgt daraus, dass der Akteur einer von ihm definierten Gruppe von Menschen spezifische normative Einstellungen zuschreibt. Das Definiens kann hierbei Verhaltensweisen beinhalten, die im Modell der Kommunikation als Kontoführung zu einer positiven oder negativen Veränderung des Punktestandes im normativen Status des Akteurs führen. Das Definiendum können wir als Typologie im Sinne einer charakterisierenden, qualitativen Zuerkennung von normativen Einstellungen - seit Pierre Bourdieu zumeist als Lebensstil gekennzeichnet - an eine vom Akteur imaginierte Gruppe von Menschen beschreiben (vgl. Welter 2006: 113f.). Diese Zuerkennung einer normativen Einstellung führt den Akteur zur Bildung von sozialen Milieus (vgl. Hradil 2006), denen er sich im Fall kongruenter normativer Einstellungen zugehörig fühlt, beziehungsweise denen er sich im Falle differenter normativer Einstellungen dementsprechend nicht zugehörig fühlt. Insofern kann man die normativen Einstellungen, die sich aufgrund kommunikativ erzeugter Punktestandsveränderungen in der Kontoführungs-Praxis der Akteure bilden, als Ausgangspunkt für soziale und kulturelle Inklusions- oder Exklusionstendenzen verstehen und konkreter als Resultat von Kommunikationsakten rekonstruieren.

\section{Image und Reputation in der Unternehmenskommunikation}

Diese Kommunikationsakte erzeugen zumeist Symbole, die auf den normativen Status der Rezipienten einwirken. Die in der Unternehmenskommunikation erzeugten Symbole, wie Schlüsselbegriffe, Farben, in der Werbung dargestellte Typen und Handlungssituationen, werden vom Rezipienten wahrgenommen und bewirken vor dem Hintergrund seiner normativen Einstellungen eine positive oder negative Veränderung jenes Kontostands, den der Akteur für den Absender der Kommunikation führt. Die Verbindung zwischen dem Unternehmen und seinen Bezugsgruppen ist ein deontischer Status, wobei jedoch nur die Rezipienten als natürliche Personen innerhalb der Bezugsgruppen über einen konkreten normativen Status verfügen. Das Unternehmen als Organisation spielt hierbei eine Sonderrolle als Kommunikator ohne normativen Status - gleichwohl es Gegenstand der Kontoführung seitens der Rezipienten ist.

Ein Image kann man insofern innerhalb des Inferentialismus als normativen Status auffassen, der sich durch zwei Antezedenzien herausbildet: Zum einen bewirken alle Akte der wahrgenommenen Unternehmenskommunikation eine positive oder negative Veränderung im Kontostand, den der Rezipient für die Organisation führt, und generieren damit einen konkreten normativen Status. Zum anderen wirken die normativen Einstellungen, die den Rezipienten mit inklusions- oder exklusionswürdigen sozialen 
Milieus verbinden, auf seinen normativen Status ein und beeinflussen damit wiederum den Punktestand.

Die Reputation eines Unternehmens ist folglich die zuerkannte normative Einstellung des Rezipienten zur Organisation und spiegelt folglich einerseits den akteurgebundenen und aktuellen normativen Status, andererseits die voluntative Zugehörigkeit beziehungsweise Ablehnung des Rezipienten mit jenem sozialen Milieu, das er mit der spezifischen normativen Einstellung verknüpft.

Image und Reputation verweisen dementsprechend wechselseitig aufeinander, ebenso wie normativer Status und normative Einstellung. Der nominalistische Standpunkt des Inferentialismus, so wie er hier formuliert wurde, schließt damit konsequent die Reputation als „gemeinsame Einstellung“ einer Gruppe aus und vermeidet jegliche Anlehnung an idealistische Konstruktionen in der Rekonstruktion von Wirkungsmodellen der Unternehmenskommunikation.

Für die Maßnahmen der Unternehmenskommunikation folgt daraus, dass der Einsatz von Symbolen in doppelter Hinsicht geplant werden muss: Zum einen bewirken Symbole eine Veränderung im Kontostand des Rezipienten; wird das symbolische Kommunikationsangebot als positiv empfunden, erhöht dies den Punktestand im deontischen Konto, das der Rezipient für das Unternehmen führt. Solche Kommunikationsangebote können attraktive Preise, Service- und Warenangebote sein; die Kommunikation findet hierbei zumeist im Raum der Gründe statt, die Sprechakte der Unternehmenskommunikation sind in diesen Fällen deskriptiver Art.

Zum anderen entfalten die Symbole ihre kommunikative Macht über die Zuerkennung normativer Einstellungen, die den Rezipienten mit positiv assoziierten Gemeinschaften verbinden. Wenn wir davon ausgehen, dass Individuen grundsätzlich ein starkes Interesse daran haben, als Teil einer Gemeinschaft zu gelten und sich gegenüber anderen Gruppen abzugrenzen - eine Annahme, die in der Sozial- und Kommunikationswissenschaft überaus gängig ist, beispielsweise in der Theorie der Schweigespirale oder in der Theorie der sozialen Identität -, um damit ihre personale und soziale Identität zu sichern, findet die Kommunikation außerhalb des deskriptiven Raums der Gründe statt, die Sprechakte sind hierbei zumeist normativer Natur.

An dieser Stelle muss man mit Herbert Schnädelbach (Schnädelbach 2004: 165) und Jürgen Habermas (Habermas 2004: 179) konstatieren, dass eine Einengung auf deskriptive Sprechakte, wie Robert Brandom sie vornimmt, unzureichend für eine kommunikationswissenschaftliche Theorie ist. Kommunikation im Sinne der Bedeutungsvermittlung zwischen Akteuren funktioniert durch deskriptive beziehungsweise konstative Sprechakte - ihre Kommunikationsmacht, die über die reine Verständigung hinausgeht, entfaltet sich aber in der Regel erst durch normative Sprechakte (vgl. Rommerskirchen 2011: 69ff.).

Dies gilt nicht zuletzt in der Unternehmenskommunikation. Günther Ortmann macht darauf aufmerksam, dass „es heutzutage Organisationen, nicht individuelle Akteure sind, die mit der mächtigsten Stimme sprechen können“ (Ortmann 2011: 372). Auch für ihn liegt die Kommunikationsmacht der Unternehmen in den performativen Effekten, in ihrer Kommunikations- und Exkommunikationsmacht, die sie über Identitätsstiftung ausüben: „Fabrikation von Identität, das meint heute vor allem die organisierte Produktion von Identität im Sinne eines Fingierens. Organisationen stellen und legen fest, was einer oder etwas ,ist', nicht nur, aber auch nicht zuletzt mit ihrer Kommunikations- und Exkommunikationsmacht“ (Ortmann 2011: 378).

Die monetären Konsequenzen der Macht der Unternehmenskommunikation zeigt aktuell das Beispiel Facebook. Die Kommunikationsplattform wurde 2004 von Mark 
Zuckerberg veröffentlicht und hat heute, nur sieben Jahre später, mehr als 750 Millionen Menschen zu ihren Mitgliedern gemacht. Nur Indien und China haben mehr Bürger als Facebook Mitglieder. Der Jahresumsatz des Unternehmens wird für das Jahr 2010 auf zwei Milliarden US-Dollar geschätzt, der Gewinn auf 400 Millionen US-Dollar (Buchter/Rohwetter 2011). Falls Facebook im Jahr 2012 an der Börse gehandelt wird, rechnen Finanzfachleute mit einer Marktkapitalisierung von bis zu 100 Milliarden US-Dollar (Thurm 2011), dem 50-fachen des Jahresumsatzes. Zum Vergleich: Der deutsche HenkelKonzern erzielte 2011 einen Gesamtumsatz von 15 Milliarden Euro (umgerechnet 21 Milliarden US-Dollar) und einen Börsenwert von 18 Milliarden Euro (umgerechnet 25 Milliarden US-Dollar). Während der Umsatz des Henkel-Konzerns also zehnmal so hoch war wie der von Facebook, betrug sein Börsenwert nur ein Viertel der aktuell vermuteten Marktkapitalisierung des US-Unternehmens.

Das große Interesse an Aktien des Unternehmens Facebook lässt sich vor dem Hintergrund des hier Gesagten wie folgt interpretieren: Die strategische Kommunikation von Facebook zielt darauf ab, die Welt in Mitglieder (Ingroup) und Nicht-Mitglieder (Outgroup) aufzuteilen. Die normativen Status der Ingroup hat Facebook erfolgreich konstruiert: durchweg jung, sozial aktiv, technikaffin, kompetent und hochkommunikativ. Dies formt die normativen Einstellungen der Rezipienten, die Ingroup gilt für die meisten von ihnen als attraktiv. In der Folge erhöht jeder Sprechakt der Unternehmenskommunikation von Facebook sein Punktekonto bei den Rezipienten, prägt damit deren normative Status und beeinflusst ihre normativen Einstellungen.

Das Resultat ist eine Art deontischer Status zwischen den Rezipienten und dem Unternehmen. Die Beziehung zwischen beiden ist nicht die eines Unternehmens zu seinen Kunden, es geht nur unwesentlich um ein Angebot zur Befriedigung eines Konsumentenbedürfnisses. Facebook konnte die Nachfrage nach seinem Angebot gezielt steuern, weil es vor allem anderen die Reputation der Marke kontrolliert hat. Angesicht eines Umsatzes von zwei Milliarden US-Dollar und eines vermuteten Börsenwertes von 100 Milliarden US-Dollar muss man festhalten, das der Wert von Facebook das Ergebnis von Unternehmenskommunikation ist - und nicht von klassischen Unternehmensleistungen für die Volkswirtschaften der Länder, in denen Facebook-Mitglieder leben und arbeiten. Der Wert von Unternehmen, insbesondere von Dienstleistungsunternehmen wie Apple, Google und Facebook, ist heute mehr denn je das Ergebnis von Unternehmenskommunikation, durch die Images und Reputation der Akteure strategisch aufgebaut werden.

\section{Fazit}

Robert Brandoms Inferentialismus ist eine kommunikationswissenschaftliche Theorie, die eine tragfähige Verbindung zwischen Sprachphilosophie und Soziologie anbietet. Durch die Verknüpfung von intrapersonaler und interpersonaler Perspektive in seinem neopragmatischen Kommunikationsmodell bietet Brandom zahlreiche Anknüpfungspunkte für benachbarte Forschungsfelder. Ein solches Feld ist die Unternehmenskommunikation, hier trägt der Inferentialismus zu einem besseren Verständnis der zentralen Begrifflichkeiten Image und Reputation bei.

Unternehmen kommunizieren durch vielfältige Maßnahmen, beispielsweise Werbung oder Öffentlichkeitsarbeit. Mit Hilfe des Inferentialismus lässt sich die Wirkung der Kommunikationsmaßnahmen differenziert betrachten: Zum einen wirken die Maßnahmen über eine Veränderung im deontischen Konto der Rezipienten auf deren normativen Status, dies resultiert in einem entsprechenden Image des kommunizierenden Unternehmens. Hierbei gilt es zu beachten, dass Punktestände im deontischen Konto 
und damit das Image durch Kommunikation - und soziales Handeln - sowohl positiv als auch negativ verändert werden können. Steigende Mitgliederzahlen bei Facebook können zu einer positiven Änderung führen, die Berichterstattung über den Missbrauch persönlicher Daten zu einer negativen.

Zum anderen wirken die Maßnahmen der Unternehmenskommunikation - mindestens ebenso mächtig - über den Inklusions- oder Exklusionswunsch der Rezipienten auf deren normative Einstellungen. Das Resultat dieser Kommunikationsakte ist ein deontischer Status, der den Rezipienten, das von ihm imaginierte soziale Milieu und das Unternehmen als fiktiven Kommunikator über diese normativen Einstellungen miteinander verbindet. Die Reputation eines Unternehmens ist im Modell des Inferentialismus eben dieser - zumeist strategisch generierte - deontische Status, der eine hohe Bindungsfähigkeit auf die Rezipienten ausüben kann.

Die Macht der Unternehmenskommunikation liegt heute weniger in der Persuasion durch Werbung als vielmehr in strategischen Appellen an die normativen Einstellungen der Rezipienten und ihr Verlangen an die Bindung an beziehungsweise Abgrenzung von sozialen Milieus. Das Modell der deontischen Kontoführung und des Inferentialismus macht diese kommunikative Macht der Unternehmen ein wenig plausibler.

\section{Literaturverzeichnis}

Austin, John L. (1962): How to Do Things with Words. Cambridge.

Berger, Peter L.; Luckmann, Thomas [1969] (2000): Die gesellschaftliche Konstruktion der Wirklichkeit. Frankfurt a. M.

Bernays, Edward [1928] (2009): Propaganda. Die Kunst der Public Relations. Freiburg i. Br.

Brandom, Robert (2000): Expressive Vernunft. Frankfurt a. M.

Brandom, Robert (2001): Begründen und Begreifen. Frankfurt a. M.

Brandom, Robert (2008): Between Saying \& Doing. New York.

Bucher, Hans-Jürgen (2004): Journalismus als kommunikatives Handeln. In: Martin Löffelholz (Hg.): Theorien des Journalismus. Wiesbaden, S. 263-285.

Buchter, Heike/Rohwetter, Marcus (2011): Geschäfte unter Freunden. In: Die Zeit 03/2011, S. 21.

Buck, Matthias (2011): Im Zweifel ein Veto. Banken müssen die Risiken für ihre Reputation ernst nehmen. In: Die Zeit 33/2011, S. 27.

Coulter, Jeff (1979): The Social Construction of Mind. London.

Cooley, Charles Horton (1909): Social Organization. New York.

Grice, Paul (1957): Meaning. In: The Philosophical Review 66, S. 377-388.

Habermas, Jürgen (1981) Theorie des kommunikativen Handelns. Frankfurt a. M.

Habermas, Jürgen (2004): Von Kant zu Hegel. Zu Robert Brandoms Sprachpragmatik. In: ders.: Wahrheit und Rechtfertigung. Frankfurt a. M., S. 138-185.

Horvarth, P.; Möller, K. (2004): Intangibles in der Unternehmenssteuerung. Strategien und Instrumente zur Wertsteigerung des immateriellen Kapitals. München.

Hradil, Stefan (2006): Soziale Milieus - eine praxisorientierte Forschungsperspektive. In: APuZ 44-45/2006, S. 3-10.

Kambartel, Friedrich; Stekeler-Weithofer, Pirmin (2005): Sprachphilosophie. Probleme und Methoden. Stuttgart.

Knell, Sebastian (2004): Propositionaler Gehalt und diskursive Kontoführung. Berlin/New York.

Knoblauch, Hubert; Luckmann, Thomas (2000): Gattungsanalyse. In: Uwe Flick u. a. (Hg.): Qualitative Forschung. Reinbek bei Hamburg, S. 538-546.

Lewis, David (1983): Scorekeeping in a Language Game. In: ders.: Philosophical Papers, Bd. 1. New York, S. 233-249.

Luhmann, Niklas (1984): Soziale Systeme. Frankfurt a. M.

Lull, James (2001): Culture in the Communication Age. London/New York.

Mead, George H. [1973] (2008): Geist, Identität und Gesellschaft. Frankfurt a. M.. 
Müller, Stefan (2011): Basel III - Aktueller Diskussionsstand, Auswirkungen auf die Unternehmensfinanzierung und Ausweichstrategien. http://www.haufe.de/finance/academy/müller.

Ortmann, Günther (2011): Die Kommunikations- und die Exkommunikationsmacht in und von Organisationen. In: DBW 4/2011, S. 357-380.

Pritzlaff, Tanja (2006): Entscheiden als Handeln. Frankfurt a. M.

Reichertz, Jo (2009): Kommunikationsmacht. Wiesbaden.

Rommerskirchen, Jan (2011): Prekäre Kommunikation. Wiesbaden.

Rorty, Richard (1967): Metaphilosophical Difficulties of Linguistic Philosophy. In: ders.: The Linguistic Turn. Chicago, S. 1-39.

Schmid, Beat F.; Lyczek, Boris (2008): Die Rolle der Kommunikation in der Wertschöpfung der Unternehmen. In: Miriam Meckel, Beat F. Schmid (Hg.): Unternehmenskommunikation. Wiesbaden, S. 3-150.

Schnädelbach, Herbert (2004): Sozialpragmatischer Idealismus. Bemerkungen zu Robert B. Brandoms „Expressive Vernunft“. In: Allgemeine Zeitschrift für Philosophie 29/2, S. 163-175.

Searle, John R. (1969): Speech Acts. Cambridge.

Thurm, Frieda (2011): Facebook wächst nicht mehr so schnell: In: Die Zeit 24/2011, S. 26.

Trommsdorff, Volker (2009): Konsumentenverhalten. Stuttgart.

Untergrabner, Gabriela; Ehrenhöfer, Robert (2004): Strategische Finanzierung über die Börse. In: Wilhelm Stadler (Hg.): Die neue Unternehmensführung. Frankfurt a. M., S. 211-223.

Welter, Markus (2006): Die Forschungsmethode der Typisierung. Charakteristika, Einsatzbereiche und praktische Anwendung. In: WiSt 2/2006, S. 113-116.

Wittgenstein, Ludwig [1921] (2003): Tractatus logico-philosophicus. Berlin.

Wittgenstein, Ludwig [1953] (2001): Philosophische Untersuchungen. Berlin.

Zerfaß, Ansgar (2004): Unternehmensführung und Öffentlichkeitsarbeit. Wiesbaden. 